\title{
Síndrome poliglandular autoimune tipo III: uma rara associação entre doença de graves, vitiligo e miastenia gravis
}

\author{
Autoimmune polyglandular syndrome type III: a rare association between graves' disease, \\ vitiligo and myasthenia gravis
}

Síndrome poliglandular autoinmune tipo III: una rara asociación entre enfermedad de graves, vitiligo y miastenia gravis

Jamila Vaz Tavares ${ }^{1}$, Amanda Machado Kahwage ${ }^{2}$.

\section{RESUMO}

Introdução: A sindrome poliglandular autoimune (SPA) é caracterizada pela associação de doenças autoimunes órgão-específicas endócrinas e não-endócrinas, e se classificam em quatro tipos dependendo dos órgãos afetados. A SPA tipo III é definida como associação entre doença da tireoide autoimune com outras entidades autoimunes como doenças gastrointestinais, neuromusculares, pele, etc. Detalhamento do caso: Este relato descreve o caso clínico de um paciente masculino de 22 anos, apresentando associação de Doença de Graves, Vitiligo e Miastenia Gravis diagnosticada por critérios clínicos bem estabelecidos e exames complementares como marcadores de autoimunidade e exames de imagem, são mostradas as complicações apresentadas pelo paciente, o tratamento instituído e sua evolução clínica. Discussão: Compara-se o caso descrito com os achados relatados na literatura, abordando a epidemiologia, apresentação clínica, métodos diagnósticos e tratamentos disponíveis, ressaltando a importância da investigação clínica detalhada, no intuito de realizar diagnósticos mais precoces e tratar adequadamente os pacientes.

Palavras-Chave: Síndrome Poliglandular Autoimune, Doença de Graves, Vitiligo, Miastenia Gravis.

\begin{abstract}
Introduction: Autoimmune polyglandular syndrome (APS) is characterized by the association of autoimmune, endocrine and non-endocrine organ-specific diseases, and is classified into four types depending on the affected organs. APS type III is defined as an association between autoimmune thyroid disease and other autoimmune diseases such as gastrointestinal, neuromuscular, skin, and other diseases. Case report: This report describes the clinical case of a 22-year-old male patient with Graves' Disease, Vitiligo and Myasthenia Gravis diagnosed by well-established clinical criteria and complementary tests such as autoimmunity markers and imaging tests. complications presented by the patient, the treatment instituted and its clinical evolution. Discussion: The case described is compared with the findings reported in the literature, addressing the epidemiology, clinical presentation, diagnostic methods and available treatments, emphasizing the importance of detailed clinical investigation, in order to make earlier diagnoses and treat patients adequately.
\end{abstract}

Keywords: Autoimmune Polyglandular Syndrome, Graves' disease, Vitiligo, Myasthenia Gravis.

\footnotetext{
${ }^{1}$ Médica residente em Clínica Médica do Hospital Ophir Loyola Belém/PA. *E-mail: jamila_vaz@hotmail.com

${ }^{2}$ Médica Endocrinologista do Hospital Ophir Loyola - Belém/PA.
} 


\section{RESUMEN}

Introducción: El sindrome poliglandular autoinmune (SPA) se caracteriza por la asociación de enfermedades autoinmunes órgano-específicas endocrinas y no endocrinas, y se clasifican en cuatro tipos dependiendo de los órganos afectados. La SPA tipo III se define como asociación entre enfermedad de la tiroides autoinmune con otras entidades autoinmunes como enfermedades gastrointestinales, neuromusculares, piel, etc. Detallado del caso: En el presente estudio se describe el caso clínico de un paciente masculino de 22 años que presenta asociación de Enfermedad de Graves, Vitiligo y Miastenia Gravis diagnosticada por criterios clínicos bien establecidos y exámenes complementarios como marcadores de autoinmunidad y exámenes de imagen, las complicaciones presentadas por el paciente, el tratamiento instituido y su evolución clínica. Discusión: Se comparó el caso descrito con los hallazgos relatados en la literatura, abordando la epidemiología, presentación clínica, métodos diagnósticos y tratamientos disponibles, resaltando la importancia de la investigación clínica detallada, con el fin de realizar diagnósticos más precoces y tratar adecuadamente a los pacientes.

Palabras clave: Síndrome Poliglandular Autoinmune, Enfermedad de Graves, Vitíligo, Miastenia Gravis.

\section{INTRODUÇÃO}

As síndromes poliglandulares autoimunes (SPA) se definem como a coexistência de uma ou mais alterações primárias das glândulas endócrinas cuja natureza é autoimune e está associada a outras patologias imunológicas com a presença de anticorpos circulantes dirigidos contra órgãos específicos. Compõem patologias de espectros variados, de maneira geral mais comuns em mulheres de meia idade, sem predileção por etnia. As SPA diferem consideravelmente quanto à idade de apresentação, o que nos permite dividi-las em dois subtipos principais: a juvenil SPA tipo I (SPA I), que ocorre por meio de uma herança monogenética, e a SPA do adulto, na qual vários genes contribuem para sua etiopatogenia, e que ainda subdivide-se em SPA II, III, e IV, a depender da combinação de doenças autoimunes. (VILAR et al, 2016; CARTAS et al, 2014; NAVARRETE-TAPIA, 2013).

A SPA tipo I se caracteriza pela presença de pelo menos duas das seguintes situações: Doença de Addison, Hipoparatireoidismo crônico e Candidíase crônica; a SPA tipo II se apresenta como associação de Doença de Addison à uma doença autoimune da tireoide e/ou diabetes mellitus tipo 1 (DM1); a SPA tipo III representa a associação de doença tireoidiana autoimune com outras doenças autoimunes como DM 1 , gastrite atrófica, vitiligo, alopecia e miastenia gravis, excluindo-se Doença de Addison e/ou Hipoparatireoidismo; e a SPA tipo IV é marcada pela combinação de doenças autoimunes que não fecham critérios para compor as categorias já mencionadas (SOUSA et al, 2015).

A SPA tipo III é definida como associação entre doença autoimune da tiroide com outras entidades autoimunes, e que de acordo com a reclassificação de Betterle pode ser subdividida em: SPA tipo Illa - doença tireoidiana associada a doenças endocrinológicas como DM1, síndrome de Mirata, hipofisite linfocítica; SPA tipo IIlb - doença da tireoide associada a doenças gastrointestinais ou hepáticas como gastrite atrófica, doença celíaca, doença inflamatória intestinal, hepatite autoimune, cirrose biliar primária; SPA tipo IIIc - doença da tireoide associada à doença da pele, neuromuscular ou hematológica como o vitiligo, alopecia, miastenia gravis, esclerose múltipla, anemia hemolítica autoimune e síndrome do anticorpo antifosfolípide; SPA tipo Illd - doença da tireoide associada doenças do colágeno e vasculite como lúpus eritematoso sistêmico, artrite reumatoide, esclerodermia (NAVARRETE-TAPIA, 2013).

Neste artigo será apresentado o relato de caso de um paciente masculino, de 22 anos, com síndrome poliglandular autoimune tipo IIlc, com a rara associação de Doença de Graves, Vitiligo e Miastenia Gravis, descrevendo o diagnóstico, tratamento e evolução clínica. 


\section{DETALHAMENTO DO CASO}

Paciente A.C.L., sexo masculino, 22 anos, caucasiano, natural e residente em Belém-PA, evoluindo durante um mês com hiperemia conjuntival, exoftalmia, ptose palpebral, edema periorbitário, taquicardia, fraqueza muscular proximal progressiva, perda de peso e mal-estar (Figura 2). Procurou atendimento médico, após o qual realizou ultrassonografia de tireoide, que evidenciava tireodeopatia difusa, e dosagens de TSH e T4 livre alteradas (Tabela 1), sendo então medicado com Metimazol $80 \mathrm{mg}$ ao dia. Nos antecedentes a família relatava aparecimento de manchas acrômicas em face, membros superiores e inferiores desde os 13 anos diagnosticadas por dermatologista como vitiligo (Figura 1).

Apesar do tratamento, manteve sintomas e procurou hospital de emergência devido dispneia. Foi medicado com sintomáticos e recebeu alta. Após 5 dias, retornou ao mesmo hospital com quadro de insuficiência respiratória aguda súbita e grave, com necessidade de intubação orotraqueal e ventilação mecânica, sendo posteriormente transferido para a unidade de terapia intensiva (UTI) do Hospital Jean Bitar. $\mathrm{Na}$ admissão hospitalar apresentava-se sedado, intubado, emagrecido, com aumento do volume da tireoide e sepse de foco pulmonar.

No $1^{\circ}$ dia de internação apresentava taquicardia e hipertensão. Foi avaliado pela equipe de endocrinologia que introduziu Propranolol $80 \mathrm{mg}$ ao dia e Hidrocortisona $300 \mathrm{mg}$ ao dia, e manteve Metimazol $80 \mathrm{mg}$ ao dia, além de solicitar hormônios tireoidianos e dosagem auto anticorpos contra tireoide (resultados na Tabela 1).

Figura 1: Sinais de Vitiligo - Machas acrômicas disseminadas pela face, membros e tronco em região anterior $(A)$ e posterior $(B)$.
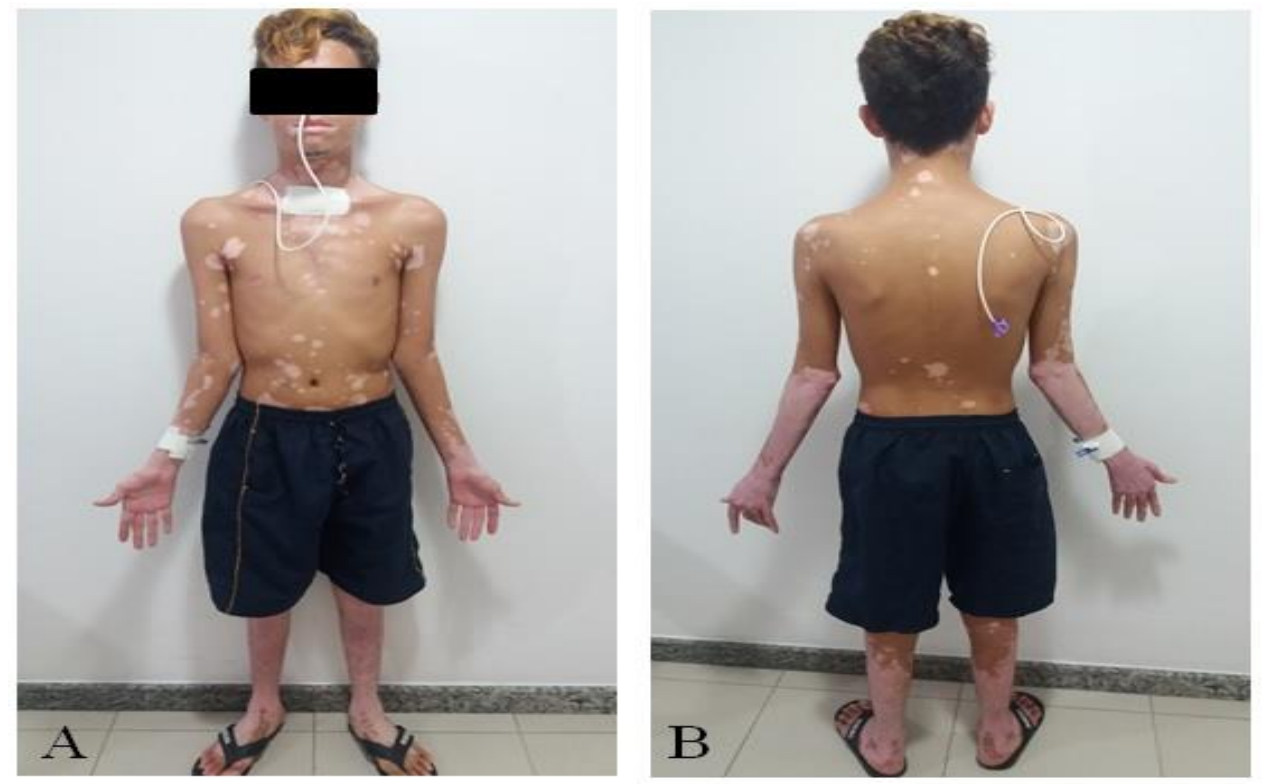

Fonte: Dados da pesquisa, 2017.

Figura 2 - Sinais de oftalmopatia da Doença de Graves exoftalmia, ptose palpebral e edema periorbitário.

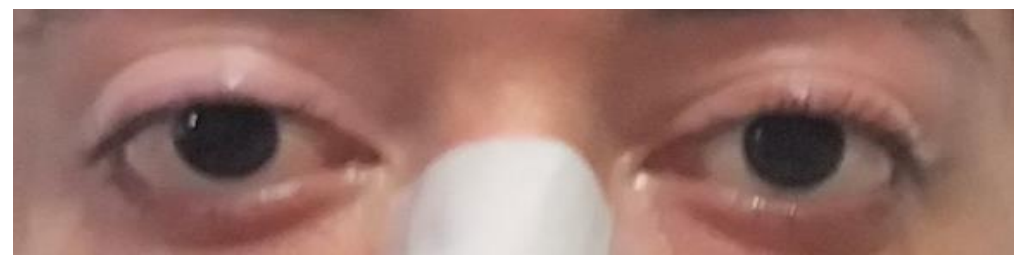

Fonte: Dados da pesquisa, 2017. 
Tabela 1 - Exames laboratoriais realizados pelo paciente durante a investigação diagnóstica.

\begin{tabular}{lcccc}
\hline Datas & $\mathbf{2 5 / 0 7 / 2 0 1 7}$ & $\mathbf{0 9} / \mathbf{0 8} / \mathbf{2 0 1 7}$ & $\mathbf{1 9 / 0 9 / 2 0 1 7}$ & Valores de Referências \\
\hline TSH & $<0,02 \mathrm{mcgUl} / \mathrm{ml}$ & $<0,01 \mathrm{mcgUl} / \mathrm{ml}$ & - & $0,4-4,5 \mathrm{mcgUl} / \mathrm{ml}$ \\
T4L & $5,49 \mathrm{ng} / \mathrm{dl}$ & $3,07 \mathrm{ng} / \mathrm{dl}$ & $2,48 \mathrm{ng} / \mathrm{dl}$ & $0,74-1,8 \mathrm{ng} / \mathrm{dl}$ \\
T3 & - & $1,10 \mathrm{ng} / \mathrm{dl}$ & - & $1,08-3,14 \mathrm{ng} / \mathrm{dl}$ \\
TRAB & - & $4,16 \mathrm{Ul} / \mathrm{L}$ & - & $\leq 0,55 \mathrm{UI} / \mathrm{L}$ \\
ANTI-TPO & - & $31,9 \mathrm{Ul} / \mathrm{ml}$ & - & $\leq 15 \mathrm{Ul} / \mathrm{ml}$ \\
AARACh & - & - & $<0,20 \mathrm{nmol} / \mathrm{l}$ & $\leq 0,25 \mathrm{nmol} / \mathrm{L}$ \\
\hline
\end{tabular}

Legenda: TSH: Hormônio Tireoestimulante; T4L: Tiroxina Livre; T3: Triiodotironina; TRAB: Anticorpo Antireceptor de TSH; Anti-TPO: Anticorpo Anti-Tireoperoxidase; AARACH: Anticorpo Anti-receptor de Acetilcolina.

Fonte: Arquivo Hospital Jean Bitar, Belém-PA, 2017.

No $4^{\circ}$ dia de internação evoluiu com parada cardiorrespiratória em ritmo de assistolia, secundária a distúrbio hidroeletrolítico e ácido básico, revertida após um ciclo de reanimação cardiopulmonar e tratamento da causa. Posteriormente foi submetido à traqueostomia, aumentada dose de Metimazol para $100 \mathrm{mg}$ ao dia e reduzida dose de Propranolol. Ao longo dos dias melhorou o nível de consciência, progrediu desmame ventilatório, com controle clínico e metabólico, recebendo alta para a enfermaria no $20^{\circ}$ dia de internação hospitalar. Apesar das medicações adequadas, mantinha fraqueza muscular proximal em membros superiores e inferiores e aparelho fonoarticulatório deficitário, que não se justificava apenas pelo hipertireoidismo e nem por miopatia do paciente crítico, surgindo então a hipótese diagnóstica de miastenia gravis associada à doença de graves e vitiligo, configurando uma síndrome poliglandular autoimune.

Foi avaliado por neurologista que sugeriu teste terapêutico com Piridostigmina $30 \mathrm{mg} 3$ vezes ao dia, e indicou dosagem do anticorpo antireceptor de acetilcolina (AARACh), realização de Eletroneuromiografia (ENMG) e tomografia computadorizada (TC) de Torax. Após o início do teste terapêutico o paciente evoluiu com melhora importante e progressiva da força muscular, a TC de Tórax demonstrou hiperplasia tímica (figura 3), o anticorpo antireceptor de acetilcolina foi negativo (tabela 1), e a ENMG demonstrou disfunção da junção neuromuscular de padrão pós-sináptico, compatível com miastenia gravis. Confirmado o diagnóstico, foi aumentada a dose de Piridostigmina para $60 \mathrm{mg} 3$ vezes ao dia. O paciente evoluiu com melhora satisfatória e no $49^{\circ}$ dia de internação recebeu alta hospitalar e foi encaminhado para manter acompanhamento ambulatorial com endocrinologista e neurologista. Posteriormente realizou timectomia eletiva e manteve controle da doença.

Figura 3: Tomografia computadorizada de tórax demonstrando hiperplasia do timo nos cortes axial $(A)$ e coronal $(B)$.
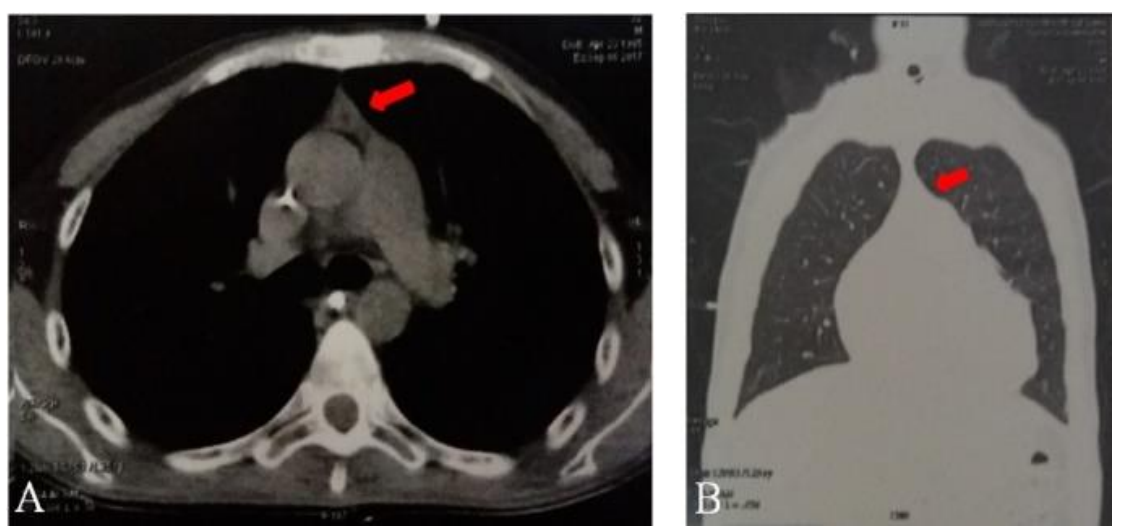

Fonte: Arquivos Hospital Jean Bitar, Belém-PA, 2017. 
Figura 4 - Eletroneuromiografia (ENMG) com teste de estimulação repetitiva positivo na placa motora do nervo acessório direito, devido decremento $>10 \%$ do $2^{\circ}$ ao $10^{\circ}$ estímulo (em vermelho).

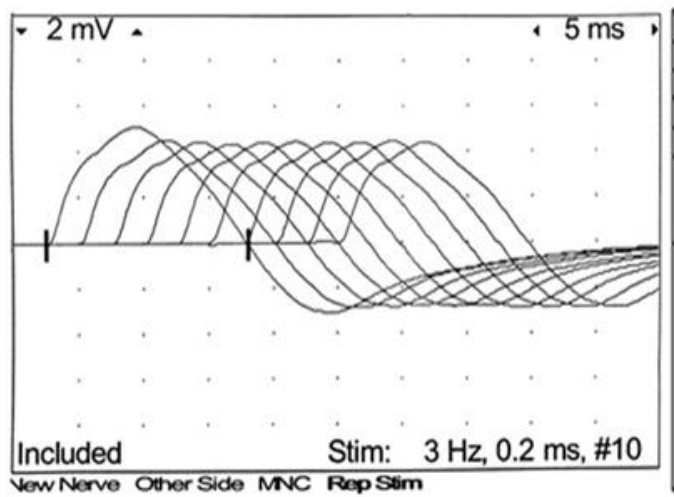

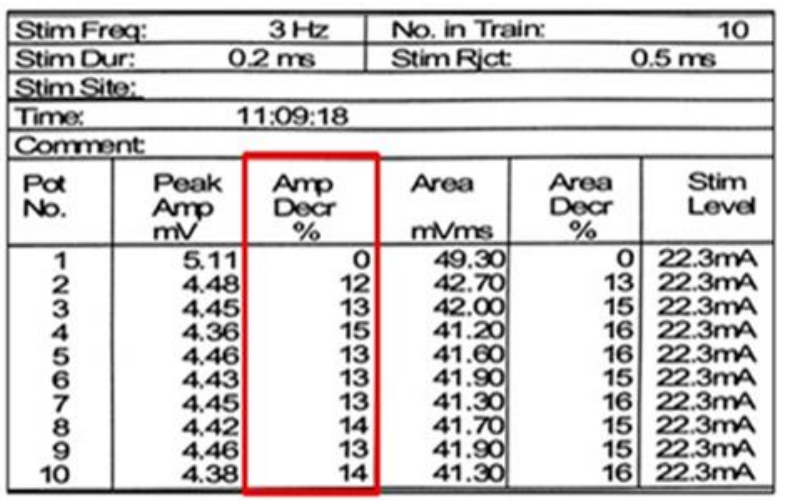

Fonte: Arquivos Hospital Jean Bitar, Belém-PA, 2017.

\section{DISCUSSÃO}

A síndrome poliglandular autoimune tipo III é o subtipo mais frequente das SPA, com uma prevalência em torno de $40 \%$, e incidência anual de 1 a 2:100.000, sendo o tipo Illa o mais comum (coexistência de diabetes melitus tipo 1 e doença autoimune tireoidiana), e tipicamente encontrada em pacientes adultos, do sexo feminino. No caso descrito o paciente era do sexo masculino, adulto jovem, com diagnóstico comprovado de doença de graves, vitiligo e miastenia gravis, caracterizando uma SPA tipo IIIc, que é menos comumente encontrada. Comparado com casos clínicos semelhantes, o perfil do paciente e a apresentação clínica, se diferem da maioria dos casos descritos na literatura, e tal associação de doenças encontrada na prática clínica se mostrou ainda mais rara comparado aos outros tipos de associação de doenças autoimunes das SPA tipo III (VILAR, et al., 2016; EIRA, et. al. 2017).

A Doença de Graves (DG) descrita por Robert Graves em 1835, é uma doença autoimune da tireoide que em sua patogênese envolve a formação do anticorpo anti-receptor do hormonio tireoestimulante (TRAb), um anticorpo que se liga aos receptores do hormônio tireoestimulante (TSH), presentes na membrana da célula folicular tireoidiana, causando, assim, a hiperfunção glandular. Clinicamente se caracteriza pela presença de hipertiroidismo, bócio, oftalmopatia e, ocasionalmente, dermopatia infiltrativa ou mixedema pré-tibial. A DG é responsável por 60-80\% dos casos de hipertireoidismo, e surge numa relação feminino/masculino de 5-10:1. Pode surgir em qualquer idade, contudo a idade típica situa-se entre os 20 e 40 anos. A prevalência é semelhante entre caucasianos e asiáticos e é menor na raça negra. As manifestações clínicas dividem-se nas que são comuns a qualquer forma de hipertireoidismo (palpitações, tremor de extremidades, perda de peso) e nas específicas para doença de graves (ptose palpebral, exoftalmia, bócio difuso). A determinação dos níveis de TSH é o método mais sensível para diagnóstico de tireotoxicose, complementada pela fração livre da tiroxina (T4L). No hipertireoidismo franco, tanto o T4L quanto a triiodotironina (T3) estão aumentados e o TSH está suprimido. A dosagem do TRAb raramente é necessária para o diagnóstico de Graves, sendo indicada apenas em casos selecionados. Apesar de ser mais típico da tireoidite de Hashimoto, o anticorpo anti-tireoperoxidase (ANTI-TPO) também pode estar presente na doença de graves. O excesso de hormônios tireoidianos secundário ao Graves pode ser controlado pela inibição da síntese hormonal, utilizando-se drogas antitireoidianas (DAT), destruição de tecido tireoidiano com iodo radioativo ((131) ou tireoidectomia total (TT).

O tratamento com betabloqueadores deve ser considerado em pacientes sintomáticos, para controle de sintomas adrenérgicos. No caso clínico, o paciente era um homem jovem, caucasiano, que abriu o quadro clínico com tireotoxicose e manifestações de DG, como fraqueza muscular, ptose palpebral, paralisia dos músculos oftalmológicos, que se confundiam com as manifestações de miastenia gravis, diferindo também em parte dos casos descritos na literatura, mais comuns em mulheres e com manifestações da doença da tireoide marcadas por hipotireoidismo, quando associadas a miastenia gravis. Durante a avaliação, foi 
submetido à dosagem dos hormônios tireoidianos e autoanticorpos que confirmaram o diagnóstico de tireotoxicose secundária a Graves. Foi então instituído tratamento com Propranolol e Metimazol, evoluindo satisfatoriamente com minimização progressiva dos sinais e sintomas. (MAIA et. al., 2013; NEVES et. al., 2008).

O vitiligo é um distúrbio pigmentar adquirido, crônico, autoimune, caracterizado pelo surgimento de manchas acrômicas em áreas de pele e mucosas, com tendência a aumentar centrifugamente de tamanho, em decorrência da ausência de melanina devido à perda progressiva de melanócitos cutâneos e a uma anormalidade em sua função. Vários fatores têm sido associados à etiopatogenia da doença, os principais são: a herança - o fator genético presente é autossômico, dominante ou recessivo e multifatorial, ou seja, com provável participação de vários genes; a autoimunidade - devido à associação positiva com algumas doenças autoimunes, e por algumas vezes compor síndromes de poliendocrinopatia; e fatores ambientais - já que 10 a $76 \%$ dos pacientes com vitiligo atribuem a doença a algum fator precipitante. O vitiligo atinge de 0,5 a $2 \%$ da população mundial. A prevalência da doença varia consideravelmente entre os diferentes grupos étnicos. Geralmente, inicia-se na infância ou em adultos jovens, e é mais comum em mulheres, apesar de muitos estudos sugerir prevalência igual entre os sexos. O paciente do caso descrito era homem, adulto jovem, que apresentou os primeiros sinais da doença aos 13 anos de idade, e progressivamente evoluiu com manchas acrômicas disseminadas pelo corpo. Esse perfil se assemelha ao perfil dos pacientes de outros casos descritos na literatura. (GIANFALDONI, et. al., 2018; ARGENTINA, 2015; DA LUZ, 2014; STEIRNER, 2004)

A Miastenia gravis (MG) é uma doença autoimune da porção pós-sináptica da junção neuromuscular (JNM), que leva a uma desordem da transmissão neuromuscular, pela ligação de autoanticorpos contra o receptor de acetilcolina (AChR) na JNM, entretanto a maneira como se inicia a produção de anticorpos é desconhecida. Existe a hipótese de que o timo esteja relacionado com a MG através da presença de um antígeno que transforma linfócitos normais em linfócitos imunocompetentes, visto que $75 \%$ dos pacientes com esta doença apresentam anormalidades no timo, tais como hiperplasia tímica e timoma. A doença muitas vezes remite após a timectomia. Recentemente se descobriu que os autoanticorpos dirigidos contra o receptor de tirosina-quinase músculo específico (MuSK) também tem um papel importante no desenvolvimento da doença. Os autoanticorpos estão presentes em $80 \%$ dos pacientes, e a doença pode ser classificada em: soropositiva e soronegativa. A incidência da miastenia varia de 1-9 por milhão de habitantes, e a prevalência de 25-142 por milhão de habitantes, havendo discreto predomínio em mulheres. A idade de início é bimodal, sendo os picos de ocorrência em torno de 20-34 anos para mulheres e 70-75 anos para homens. A MG é caracterizada clinicamente por fraqueza flutuante que melhora com o repouso e piora com o exercício, infecções, menstruação, ansiedade, estresse emocional e gravidez. A fraqueza pode ser limitada a grupos musculares específicos (músculos oculares, faciais) ou ser generalizada em que a fraqueza afeta tanto os músculos oculares quanto as funções bulbares (disartria, disfagia), músculos dos membros ou músculos respiratórios. Pode ocorrer a chamada crise miastênica, que é definida por insuficiência respiratória associada à fraqueza muscular grave. O diagnóstico é baseado em manifestações clínicas, dosagem de autoanticorpos e estudos eletrofisiológicos, como a eletroneuromiografia (ENMG). O tratamento inicial consiste no uso de inibidores da acetilcolinesterase (AChE), como a piridostigmina. Entretanto, estas drogas isoladamente não costumam controlar a doença, sendo preciso associação com outros métodos como imunossupressor, timectomia ou altas doses de corticosteroides. O paciente do estudo apresentou a forma generalizada de MG, com fraqueza tanto nos músculos oculares, como de membros superiores e inferiores, alterações de deglutição, de fala e até insuficiência respiratória aguda, caracterizando uma crise miastênica. $\mathrm{Na}$ investigação, foi dosado o autoanticorpo AChR com resultado negativo, porém o MuSK não foi testado por não estar disponível. Foi também realizada ainda tomografia computadorizada do tórax que identificou a presença de hiperplasia tímica; e a eletroneuromiografia demonstrou teste de estimulação repetitiva positivo nas placas motoras dos nervos facial e acessório direito, com decremento maior que $10 \%$ do $2^{\circ}$ ao $10^{\circ}$ tempo do teste, padrão compatível com a doença. O paciente então mesmo recebeu uma dose inicial de piridostigmina ( $30 \mathrm{mg} 3 \mathrm{x} / \mathrm{dia}$ ) e evoluiu com melhora progressiva da força muscular, sendo posteriormente aumentada a dose e foi submetido a timectomia proporcionando estabilidade clínica da doença (TORRE, et al., 2018; SANDERS et al., 2016; DE MARCHI, 2015; DUMAN et. al., 2014; BRANCO et al., 2011). 
Associação de doenças autoimunes como as descritas acima e encontradas nesse caso clínico reforçam a necessidade de sempre pensar que indivíduos portadores de algum grau de autoimunidade têm maior risco que a população geral, de desenvolver outras doenças autoimunes. Os estudos demonstram que raramente estão presentes várias doenças autoimunes na apresentação inicial do quadro clínico, podendo haver diferença de vários anos entre um diagnóstico e outro, como ocorreu no caso descrito em que as manifestações de doença autoimune da pele ocorreram na adolescência e as de doença autoimune da tireoide e neuromuscular, ocorreram anos depois. Considera-se que uma síndrome poliglandular é potencial quando pelo menos uma das patologias indispensáveis ao diagnóstico se encontra em uma fase pré-clínica (com marcadores imunológicos positivos, mas ainda sem disfunção confirmada pelo estudo complementar), porém ainda há controvérsia quanto à real necessidade de rastreio de outras patologias autoimunes, diante de um diagnóstico realizado. Algo que os autores são unânimes em afirmar, é que quanto mais cedo a síndrome é diagnosticada, mais precoce é o tratamento e melhor será o prognóstico do paciente. Afinal, como no caso descrito, o paciente já havia recebido diagnóstico de hipertireoidismo, iniciado tratamento antes de evoluir com sintomas motores e respiratórios. Se durante a internação não se houvesse pensado na associação de miastenia gravis a doença de graves, o paciente poderia ter recebido alta hospitalar, e posteriormente retornado em nova crise miastênica, talvez com um quadro ainda mais grave que o anterior, e com altas possibilidades de óbito. Portanto, enfatiza-se a necessidade de um olhar clínico atento para identificar sinais e sintomas de doença autoimune associadas, a busca por realizar o diagnóstico o mais rápido possível e iniciar prontamente o tratamento, a fim de que esses pacientes tenham boa evolução clínica e qualidade de vida. (EIRA, et. al., 2017; GOLVEIA et. al.; 2013)

Enfatiza-se por último, que os estudos a respeito de tal patologia e descrições de casos clínicos ainda são limitados na literatura nacional, sendo de origem internacional a maioria dos artigos encontrados nas bases de dados pesquisadas, demonstrando a necessidade de fomentar a pesquisa sobre o tema em nosso país a fim de obter dados mais consistentes a respeito da incidência, prevalência e dados clínicos da SPA na população brasileira, no intuito de diagnosticar e tratar de maneira mais efetiva os pacientes.

\section{REFERÊNCIAS}

1. ARGENTINA. Dermatologia. Sociedade Argentina de Dermatologia. Consenso sobre Vitiligo. Argentina, 2015.

2. BRANCO, ACSC et al. Atualizações e Perspectivas na Miastenia gravis. Revista Brasileira de Ciências da Saúde, 2011; 15(4): 493506.

3. CARTAS, US et al. Síndrome Poliglandular tipo III. A propósito de un caso. Revista Cubana de Reumatología, 2014; 16(3): 400-403.

4. CHAPARRO, JMO et. al. Síndrome poliglandular autoinmune tipo IIIC: reporte de un caso. Rev Esp Geriatr Gerontol, 2014; 49(5): 243-247.

5. DA LUZ, LL; DOS SANTOS, SL; PARTATA, AK et al. Vitiligo e Seu Tratamento. Revista Científica do ITPAC, Araguaína, 2014; 7(3).

6. DE MARCHI, SU; CECCHIN, E; DE MARCHI, S et al. Autoimmune spontaneous chronic urticaria and generalized myasthenia gravis in a patient with polyglandular autoimmune syndrome type 3. Muscle \& nerve, 2015; 52(3): 440-444.

7. EIRA, C. et. al. Síndrome Poliglandular Autoimune Tipo III. Revista Portuguesa de Endocrinologia, Diabetes e Metabolismo, 2017; 2(12): 200-205.

8. GIANFALDONI, S et. al. Vitiligo in Children: A Review of Conventional Treatments. Open Access Maced J Med Sci, 2018; 1(6): 213217.

9. GOUVEIA, S et al. Rastreio de síndrome poliglandular autoimune em uma população de pacientes com diabetes melito tipo 1. Arq Bras Endocrinol Metab., 2013; 57(9): 733-738.

10. MAIA, AL et al. Consenso brasileiro para o diagnóstico e tratamento do hipertireoidismo: recomendações do Departamento de Tireoide da Sociedade Brasileira de Endocrinologia e Metabologia. Arq Bras Endocrinol Metab., 2013; 57(3): 205-232.

11. NAVARRETE-TAPIA, U. Síndrome poliglandular autoinmune. Rev Med Hosp Gen Méx., 2013; 76(3).

12. NEVES, C et. al. Doença de Graves. Arquivos de Medicina, 2008; 22(4/5): 137-146.

13. RODRÍGUEZ, FJ et al. Síndrome Poliglandular Autoinmune tipo III, Reporte de un caso. Honduras Pediátrica, 1997; 18,(2): 44-46.

14. SANDERS, DB et al. International consensus guidance for management of myasthenia gravis. Neurology, 2016; 87(4): 419-425.

15. SOUSA, AWP et al. Polyglandular Syndrome Type III and Severe Peripheral Neuropathy: An Unusual Association. GE Port J Gastroenterol,, 2015; 22(1): 15-18.

16. STEINER, D et al. Vitiligo. An. Bras. Dermatol,, Rio de Janeiro, 2004; 79(3): 335-351.

17. TORRE, SM; MOLINERO, IG; GIRÓN, RM et al. Puesta al día en la miastenia gravis. Semergen, 2018.

18. UNGERER, M et al. Review of Mouse Models of Graves' Disease and Orbitopathy-Novel Treatment by Induction of Tolerance. Clinic Rev Allerg Immunol., 2016; 2(52): 182-193.

19. VILAR, L et al. Endocrinologia clínica 6. ed. Rio de Janeiro: Guanabara Koogan, 2016; 1555-1566p. 